\title{
How the Charge-Neutrality Level of Interface States Controls Energy Level Alignment in Cathode Contacts of Organic Bulk-Heterojunction Solar Cells
}

\author{
Antonio Guerrero, ${ }^{\dagger}$ Luís F. Marchesi, ${ }^{,+\ddagger}$ Pablo P. Boix, $^{\dagger}$ Sonia Ruiz-Raga, ${ }^{\dagger}$ Teresa Ripolles-Sanchis, ${ }^{\dagger}$ \\ Germà Garcia-Belmonte, ${ }^{\dagger, *}$ and Juan Bisquert ${ }^{\dagger}$
}

†Photovoltaic and Optoelectronic Devices Group, Departament de Física, Universitat Jaume I, ES-12071 Castelló, Spain and

†Laboratório Interdisciplinar de Eletroquímica e Cerâmica (LIEC), Universidade Federal de São Carlos, São Carlos, Brazil

I n organic photovoltaic devices, outer interface structures play a significant role in establishing optimal contact conditions for efficient extraction (or blocking) of charge carriers. Buffer layers of different nature are currently employed to enhance both power conversion efficiency (PCE) and cell stability by improving contact performance. Several materials have been explored to enhance the electron selectivity of the cathode contact: alkali metal compounds ( $\mathrm{LiF}$, etc.), metal oxides $\left(\mathrm{TiO}_{x}, \mathrm{ZnO}\right.$, etc.), and low molecular weight organic compounds have been reported to contribute to the overall PCE and solar cell lifetime, as reviewed in recent reports. ${ }^{1-3}$ Among those approaches, the effect of the dipole moment associated with self-assembled monolayers (SAM) attached to the interface, which alter the energy level alignment between the cathode metal and the bulk of the blend, ${ }^{4}$ is particularly interesting, as well as the inclusion of conjugated polyelectrolyte interlayers. ${ }^{5}$ In all of these cases, the energy shift induced by the charge dipole built up at interface layers enables the use of air-stable high work function metals. It is then inferred that electrostatic mechanisms occurring at the nanometer scale, both in the active layer bulk and at interfaces, have a great influence on the overall device operation. ${ }^{6,7}$

Interface dipole layers are regarded as a determining ingredient of the metal/organic contact equilibration. ${ }^{8-12}$ Several models have been proposed to account for the energy level alignment at interfaces, depending on the degree of interaction between the metal contact and the deposited organic layer. When the chemical interaction between the metal and contacting
ABSTRACT Electronic equilibration at the metal-organic interface, leading to equalization of the Fermi levels, is a key process in organic optoelectronic devices. How the energy levels are set across the interface determines carrier extraction at the contact and also limits the achievable open-circuit voltage under illumi-
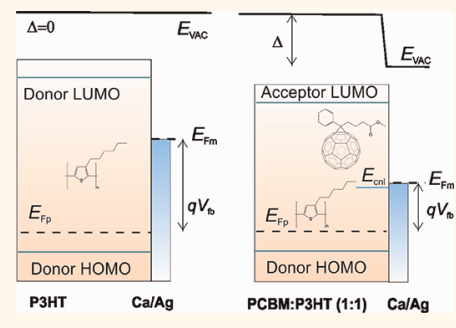
nation. Here, we report an extensive investigation of the cathode energy equilibration of organic bulk-heterojunction solar cells. We show that the potential to balance the mismatch between the cathode metal and the organic layer Fermi levels is divided into two contributions: spatially extended band bending in the organic bulk and voltage drop at the interface dipole layer caused by a net charge transfer. We scan the operation of the cathode under a varied set of conditions, using metals of different work functions in the range of $\sim 2 \mathrm{eV}$, different fullerene acceptors, and several cathode interlayers. The measurements allow us to locate the charge-neutrality level within the interface density of sates and calculate the corresponding dipole layer strength. The dipole layer withstands a large part of the total Fermi level mismatch when the polymer:fullerene blend ratio approaches $\sim 1: 1$, producing the practical alignment between the metal Fermi level and the chargeneutrality level. Origin of the interface states is linked with fullerene reduced molecules covering the metal contact. The dipole contribution, and consequently the band bending, is highly sensitive to the nature and amount of fullerene molecules forming the interface density of states. Our analysis provides a detailed picture of the evolution of the potentials in the bulk and the interface of the solar cell when forward voltage is applied or when photogeneration takes place.

KEYWORDS: metal-organic interfaces - photovoltaic devices · cathode contact $\cdot$ charge-neutrality level $\cdot$ dipole layer $\cdot$ band bending

conjugated molecules or polymers is not negligible, it is expected that molecules attached to the metal surface undergo both a shift and a broadening of their molecular energy levels. Energy distribution of the attached molecules should be modeled by a specific interfacial density of states (IDOS) which differs from that encountered in the bulk of the organic layer. The situation is @2012 American Chemical Society 
A Energy levels

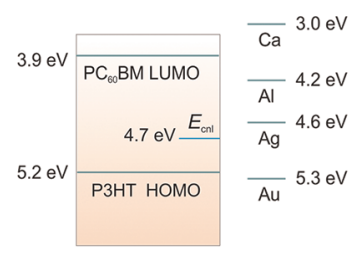

C After contact (dark) $\quad V_{\text {app }}=0$

$E_{\text {vac }}$
B Before contact $E_{\mathrm{VAC}}$

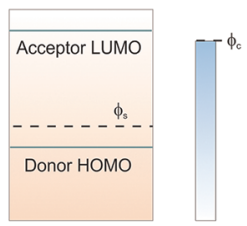

D Flat-band conditions Evac

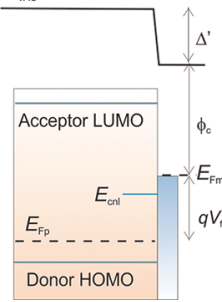

Figure 1. (A) Energy levels of organic materials and cathode metals. $E_{\mathrm{CNL}}$ signals the position of the charge-neutrality level. (B) Diagram describing the situation before the cathode contact is made: there exists a mismatch between organic and metal work functions, $\phi_{c}-\phi_{s}$, which should be equilibrated after contact. (C) In equilibrium $\left(V_{\text {app }}=0\right.$ in the dark), the vacuum level offset is split into two contributions: one arising from the bulk band bending $q V_{b b}$, the other caused by the interface dipole layer $\Delta$. A net charge transfer approaches the metal Fermi level to CNL. (D) In flatband conditions, $V_{\mathrm{app}}=V_{\mathrm{fb}}$, the dipole layer $\Delta^{\prime}$ persists in such a way that $q V_{\mathrm{fb}}=q V_{\mathrm{bb}}-\Delta+\Delta^{\prime}$.

even more featured for interfaces formed by metals deposited onto organic layers because of metal diffusion and the creation of bonding sites in a rough surface.

To rationalize these kinds of interfaces, a key parameter accounting for the interface equilibration is the charge-neutrality level (CNL) located at a given position within the IDOS. ${ }^{13-15}$ The formation of an interface dipole layer $\Delta$ whose sign depends on the energy difference, $E_{\mathrm{CNL}}-\phi_{\mathrm{C}}$, entails a charge transfer that pulls the metal Fermi level $\phi_{\mathrm{c}}$ toward $\mathrm{CNL}\left(E_{\mathrm{CNL}}\right)$. As an illustration, given the CNL location in Figure $1 \mathrm{~A}$, the metals $\mathrm{Ca}, \mathrm{Al}$, and $\mathrm{Ag}$ would transfer negative charge to the interfacial states, while Au gives rise to a positive charge sheet on the organic side. The formation of interface dipoles directly produces an offset in the vacuum level.

Despite the essential insights that the previous models have provided, a complete view of deviceintegrated metal/organic contact, including the connection with the organic bulk forming the active layer in solar cells, has not been established yet. This unified approach that integrates both metal cathode and organic bulk sides, along with interface states, is an essential advance in understanding device formation and operation and constitutes the main subject of this work.

In a real device, overall equilibration of the cathode contact should also consider the interaction with the organic layer bulk. ${ }^{16,17}$ Before contact is made, there exists an energy offset between the metal and the organic semiconductor work functions, $\phi_{c}$ and $\phi_{s}$ respectively, as schematically illustrated in Figure 1B. Donor polymers as poly-(3-hexylthiophene), P3HT can easily undergo oxidation ( $p$-doping) when exposed to the air or moisture, ${ }^{18,19}$ producing a change in the hole conductivity. ${ }^{20}$ Accordingly, we have proposed very recently that doping of the organic layer produces native mobile charges in the organic blend, which gives rise to the separate equilibration of each contact. Due to the p-doped character of the polymer, $\phi_{\mathrm{s}}$ is located approaching the donor HOMO level $\left(E_{\mathrm{HOMO}}^{\mathrm{D}} \sim\right.$ $5.2 \mathrm{eV}$ for P3HT). Whereas the cathode equilibrates the mismatch $\phi_{c}-\phi_{s}$, which is accommodated between the spatially distributed band bending within the blend layer, and the local voltage drop at the interface $\Delta$, the anode forms a good ohmic contact because of the proper energy alignment. The band bending occurs as a consequence of the hole depletion zone built up near the contact. A detailed explanation on the depletion zone formation and consequences on the device performance has been recently published. ${ }^{21}$ Experimental techniques such as Kelvin probe ${ }^{17,22}$ and ultraviolet photoelectron spectroscopy ${ }^{23}$ have given evidence of the formation of depletion zones at metal/organic contacts with the corresponding band bending. This is schematically drawn in Figure $1 C$. The voltage $V_{\mathrm{bb}}$ accounts solely for the band bending part, with $\Delta$ being caused by the presence of an interfacial dipole layer. The dipole layer exhibits a negative charge on the organic side which is compensated by a positive charge at the metal contact. Accordingly, the overall vacuum level offset can be split into two parts: one originated by the spatially extended depletion region, and the other confined at the interface dipole layer. An expression for the cathode equilibration is then written as follows

$$
q V_{\mathrm{bb}}=E_{\mathrm{F}}-\phi_{\mathrm{c}}-\Delta
$$

Here $E_{\mathrm{F}}=\phi_{\mathrm{s}}$ corresponds to the Fermi level in equilibrium.

In this work, we address the issue of electronic equilibration of the cathode contact in bulk-heterojunction solar cells by using the information extracted from an exclusively electrical technique such as the Mott-Schottky analysis of capacitance-voltage $(C-V)$ characteristics. $^{24}$ In general, capacitance in organic devices is observed to be frequency-dependent because of the presence of defect state distributions, ${ }^{25}$ however, in reverse, and low forward voltage capacitance usually exhibits a plateau at intermediate frequencies (100 Hz to $1 \mathrm{kHz}$ ), which allows for an estimation of the depletion zone width. ${ }^{24,26,27}$ A simple model is proposed here that integrates both voltage drops, namely, $q V_{\mathrm{bb}}$ and $\Delta$, into a unified description of the contact equilibration. The model is formulated in 
terms of CNL and IDOS as parameters. It is then explained how the band bending and the dipole layer strength can be readily derived from the experiment. By varying the metal used in the cathode contact, we are able to determine the CNL position which governs the dipole layer contribution through the partial IDOS occupancy. Moreover, the use of solar cells prepared from three different fullerene acceptors and the variation of the blend composition let us understand the origin and function of interface states withstanding the dipole layer voltage drop.

The model developed here provides important and detailed information of a core aspect of $\mathrm{BHJ}$ solar cell device operation, ${ }^{21}$ which is the evolution of the potentials in the bulk and the interface when either forward voltage is applied or photogeneration takes place.

\section{RESULTS AND DISCUSSION}

Capacitance-Voltage Characteristics. Solar cells of structure indium tin oxide (ITO)/poly(3,4-ethylenedioxythiophene):poly(styrene sulfonic acid) (PEDOT:PSS)/P3HT: fullerene/metal were prepared as described in the Methods section. In order to test the effect of the acceptor LUMO level shift on the cathode energetics, different fullerene acceptors have been tested, namely, indene- $C_{60}$ bisadduct (ICBA), [6,6]-phenyl- $C_{61}$-butyric acid methyl ester $\left(\mathrm{PC}_{60} \mathrm{BM}\right)$, and similar $\mathrm{C}_{71}$ derivative $\left(\mathrm{PC}_{70} \mathrm{BM}\right)$. Capacitance measurements for Mott-Schottky analysis were performed as explained in Methods. AC oscillating amplitude was as low as $20 \mathrm{mV}$ (rms) to maintain the linearity of the response, and measuring frequency was fixed in the low-frequency range $(100 \mathrm{~Hz})$ as reported in previous papers. ${ }^{24}$

An example of the measured current-density voltage $j-V$ characteristics under simulated AM1.5G illumination (1000 $\mathrm{W} \mathrm{m}^{-2}$ ) of $\mathrm{P} 3 \mathrm{HT}: \mathrm{PC}_{60} \mathrm{BM}$ solar cells using different cathode metals is plotted in Figure 2a. Relatively low power conversion efficiency (Table 1) is obtained with devices comprising a Ca cathode presumably because of the active layer thickness ( $\sim 350 \mathrm{~nm}$ ) used. We will later show the response of thinner films. Capacitance measured in the dark is drawn as a function applied voltage. The expected increase in capacitance in excess of the geometrical value $\left(C_{\mathrm{g}} \approx 10 \mathrm{nF} \mathrm{cm}{ }^{-2}\right)$ is observed (Figure $2 \mathrm{~b}$ ) because of the modulation of depletion zone by the change of voltage. ${ }^{24}$ Recent studies using different techniques support the interpretation of the capacitance rise at low forward voltages in terms of the formation of a depletion zone near the cathode contact. $^{22,28}$ By examining Figure 2c, which corresponds to Mott-Schottky plots, one can realize how the flat-band voltage $V_{\mathrm{fb}}$ extracted from the intercept value changes with the cathode metal (see Supporting Information and Table 1). We systematically observed that low work function metals such as Ca yield
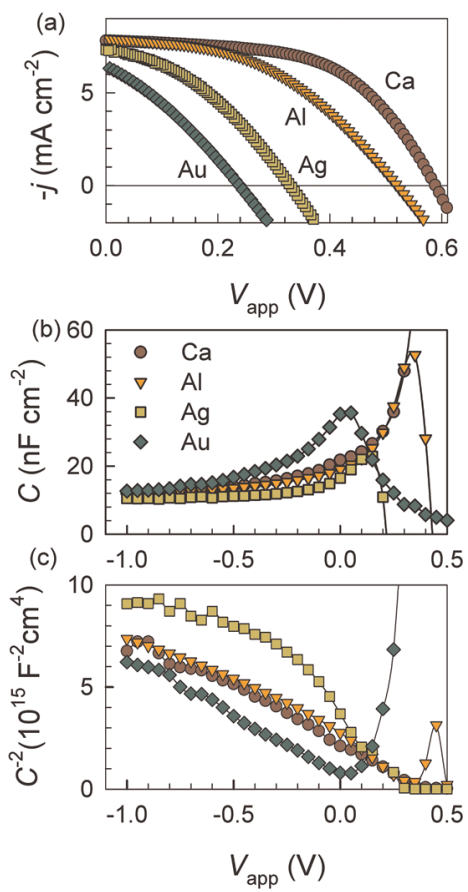

Figure 2. (A) Current-voltage characteristics of devices with structure ITO/PEDOT:PSS/P3HT:PCBM/metal. (B) Capacitance-voltage characteristics showing full depletion in reverse and the rise in capacitance toward forward voltages caused by the reduction in depletion region width. (C) Mott-Schottky plots from which the p-doping level $N$ and flat-band voltage $V_{\mathrm{fb}}$ are extracted.

TABLE 1. Photovoltaic Parameters and Parameters Extracted from the Capacitance Mott-Schottky Analysis of Thick P3HT: Fullerene Solar Cells with Different Cathode Metal

\begin{tabular}{|c|c|c|c|c|c|c|c|c|}
\hline $\begin{array}{c}\text { P3HT:PC }{ }_{60} \mathrm{BM} \\
(350 \mathrm{~nm})\end{array}$ & $\begin{array}{c}j_{\mathrm{sc}} \\
\left(\mathrm{mA} \mathrm{cm} \mathrm{cm}^{-2}\right)\end{array}$ & $\begin{array}{l}V_{\text {oc }} \\
\text { (V) }\end{array}$ & FF & $\begin{array}{l}\text { PCE } \\
(\%)\end{array}$ & $\begin{array}{c}N \\
\left(10^{15} \mathrm{~cm}^{-3}\right)\end{array}$ & $\begin{array}{l}V_{\mathrm{fb}} \\
(\mathrm{V})\end{array}$ & $\begin{array}{l}E_{\mathrm{Fm}} \\
(\mathrm{eV})\end{array}$ & $\begin{array}{c}\Delta \\
(\mathrm{eV})\end{array}$ \\
\hline $\mathrm{Ca}$ & 7.81 & 0.59 & 0.56 & 2.6 & 6.6 & 0.39 & 4.56 & 1.69 \\
\hline Al & 7.91 & 0.52 & 0.45 & 1.8 & 4.7 & 0.35 & 4.60 & 0.45 \\
\hline $\mathrm{Ag}$ & 7.36 & 0.33 & 0.30 & 1.0 & 2.5 & 0.24 & 4.71 & 0.11 \\
\hline $\mathrm{Au}$ & 6.50 & 0.24 & 0.30 & 0.5 & 7.1 & 0.14 & 4.80 & -0.49 \\
\hline
\end{tabular}

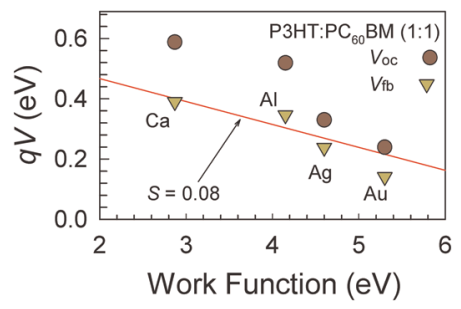

Figure 3. Experimental values of $V_{\mathrm{fb}}$ and $V_{\mathrm{oc}}$ as a function of the metal used in the cathode contact $\mathrm{Ca}, \mathrm{Al}, \mathrm{Ag}$, or $\mathrm{Au}$. While $V_{\text {oc }}$ is higher the lower the metal work function is, $V_{\mathrm{fb}}$ saturates around $\sim 0.4 \mathrm{~V}$. The slope $S$ defined in eq 7 is calculated from the linear fit considering $\mathrm{Ca}, \mathrm{Al}$, and $\mathrm{Ag}$.

higher open-circuit voltage $V_{\text {oc }}$ values (see Figure 3 ). For metals with work function higher than the fullerene LUMO level $(\sim 3.9 \mathrm{eV})$, a voltage loss appears as a consequence of the electron energy mismatch between 
the active layer and the extracting contact. The flat-band voltage resulting from the Mott-Schottky analysis follows the same trend as $V_{\text {oc }}$ for higher work function metals, as observed in Figure 3. However, in the case of Ca as the cathode contact, it is found to be $V_{\mathrm{fb}} \approx 0.4 \mathrm{~V}$, far below the $V_{\text {oc }}$ achieved. It is worth noting that, despite a large variation ( $>2 \mathrm{eV}$ ) among the metal cathode work functions, only a small change is observed for $V_{\mathrm{fb}}$ ( $\sim 0.4-0.2 \mathrm{~V}$ as listed in Table 1$)$. Accordingly the slope between $q V_{\mathrm{fb}}$ and the metal work function $\phi_{\mathrm{c}}$ is experimentally observed to be very small, $S=0.08$. Because of the reasons given below, we have left aside the case of $\mathrm{Au}$ in this slope calculation. We have already related in previous papers these low $V_{\mathrm{fb}}$ found for low work function metal values to the presence of a dipole layer made up at the organic layer/metal interface. ${ }^{21}$

Model for Determination of Flat-Band Voltage and Dipole Layer. We will next show how to calculate the flat-band voltage by considering simple electrostatic arguments. ${ }^{29,30}$ At flat-band voltage, the space charge through the depletion layer is negligible, and therefore, the interface negative charge $Q_{i s}$ is fully compensated by a positive charge at the metal $Q_{c}$, that is, $-Q_{i s}=Q_{c}$. By examining Figure 1D, one can readily express the flat-band voltage as a function of the dipole potential drop $\Delta^{\prime}$ (in flat-band conditions)

$$
q V_{\mathrm{fb}}=E_{\mathrm{Fp}}-\phi_{\mathrm{c}}-\Delta^{\prime}
$$

Here $E_{\mathrm{Fp}}$ corresponds to the hole Fermi level at forward applied voltage. We assume that $E_{\mathrm{Fp}}$ practically corresponds to the Fermi level at equilibrium (Figure 1C). The interface dipole contribution is related to the interface charge as

$$
\Delta^{\prime}=\frac{\delta Q_{\text {is }}}{\varepsilon_{\mathrm{i}} \varepsilon_{0}}
$$

where $\varepsilon_{\mathrm{i}}$ accounts for the dielectric constant of the interface layer $\left(\varepsilon_{\mathrm{i}} \sim 3\right.$ for an organic layer), $\varepsilon_{0}$ is the permittivity of the free space, and $\delta$ stands for the charge separation at the dipole layer $(\delta \sim 5 \AA)$, assuming that a molecular monolayer accommodates the interface charge.

As previously introduced, the CNL plays a determining role in the interface equilibration. In this model, specific gap states are assumed at the surface in contact with the metal. The character of the surface states changes from mostly acceptor type approaching the bottom of the conduction band to mostly donor type close to the top of the valence band. $E_{\mathrm{CNL}}$ represents the energy that marks the separation between the two classes of states within the IDOS. It is also assumed that the interface states readily achieve equilibrium with the metal electronic states. If the Fermi level of the metal is initially above $E_{\mathrm{CNL}}$, then electrons are transferred to IDOS, while the opposite is true if the Fermi level of the metal lies below CNL. The net consequence of the transference to this type of surface states distribution is that a dipole is built such that the
Fermi level of the metal will tend to align with the charge-neutrality level. The fact that different metals can be compared is due to the relatively weak sensitivity of the organic CNL to the metal/organic interaction. ${ }^{15}$ If the IDOS is very high, all metal Fermi levels will be pulled to the same place, $E_{\mathrm{CNL}}$, and the interface is said to undergo Fermi level pinning. In this case, the interfacial states are able to accommodate all of the charge required to equilibrate a large portion of offset in work functions $\phi_{\mathrm{c}}-\phi_{\mathrm{s}}{ }^{13}$ In general, the equilibration consists both of the dipole layer contribution and the band bending. ${ }^{29}$

The interface charge is obtained by assuming that the occupation of the interface state distribution IDOS, $g_{\mathrm{is}}$ is governed by the position of the metal Fermi level, $E_{\mathrm{Fm}}$

$$
Q_{\text {is }}=-q \int_{E_{\mathrm{CNL}}}^{E_{\mathrm{Fm}}} g_{\mathrm{is}} \mathrm{d} E
$$

Here, the integral extends from the charge-neutrality level to $E_{\mathrm{Fm}}$, as shown in Figure 1D. As remarked before, it is assumed that the interface states are in intimate connection (equilibrium) with the metal Fermi level through a very thin separating layer $(<10 \AA)$. The dipole layer then is transparent to electrons, while it can withstand a potential difference across it. In the specific case of organic devices, the dipole layer formed between interface organic molecules and metal contacts has been taken into account in previous models on charge injection through metal/organic interfaces. ${ }^{16,31,32}$

Since the $E_{\mathrm{Fm}}$ position depends on the applied voltage to achieve flat-band conditions, in which $E_{\mathrm{Fm}}=$ $E_{\mathrm{Fp}}-q V_{\mathrm{fb}}$, it is clear from eqs 3 and 4 that $\Delta^{\prime}$ is dependent on $q V_{\mathrm{fb}}$. In general, eq 4 should be solved numerically after considering an adequate IDOS. By assuming that $g_{\text {is }}$ varies weakly between $E_{\mathrm{CNL}}$ and $E_{\mathrm{Fm}}$, eq 4 can be approximated as $Q_{\text {is }}=q g_{\text {is }}\left(E_{\mathrm{CNL}}-E_{\mathrm{Fm}}\right)$. Equation 2 can be then rewritten as follows

$$
q V_{\mathrm{fb}}=E_{\mathrm{Fp}}-\phi_{\mathrm{c}}-\frac{q \delta g_{\mathrm{is}}}{\varepsilon_{\mathrm{i}} \varepsilon_{0}}\left(E_{\mathrm{CNL}}-E_{\mathrm{Fp}}+q V_{\mathrm{fb}}\right)
$$

After some calculations, one arrives at the expression

$$
q V_{\mathrm{fb}}=E_{\mathrm{Fp}}-E_{\mathrm{CNL}}-S\left(\phi_{\mathrm{c}}-E_{\mathrm{CNL}}\right)
$$

The parameter $S$, which accounts for the effect of $g_{\text {is }}$ on the contact energetics, is expressed as follows when a constant IDOS $g_{\text {is }}$ is assumed

$$
S=\frac{1}{1+q \delta g_{\mathrm{is}} / \varepsilon_{\mathrm{i}} \varepsilon_{0}}
$$

It is important to note that large interface density values, $g_{\text {is }} \gg$, entail $S \approx 0$ in such a way that the barrier becomes independent of the metal work function. On the contrary, $g_{\text {is }} \ll$ would imply $S \approx 1$ recovering the so-called Schottky limit. Equation 6 predicts a linear relationship between $q V_{\mathrm{fb}}$ and $\phi_{\mathrm{c}}$, with a negative slope equaling the parameter $S$. 
Strictly speaking, calculations made in flat-band conditions $V_{\mathrm{app}}=V_{\mathrm{fb}}$ (which are related to the experimental procedure to extract $V_{\mathrm{bb}}$ ) might not coincide with those occurring in equilibrium $\left(V_{\mathrm{app}}=0\right)$. In other words, parameters in eq 1 and eq 2 could differ because of the voltage dependence of the dipole contribution. This issue was discussed by Cowley, ${ }^{30}$ who arrived at the conclusion that $q V_{\mathrm{fb}}=q V_{\mathrm{bb}}-\Delta+\Delta^{\prime}$. The variation of the dipole strength $\Delta-\Delta^{\prime}$ is proportional to the factor $S\left(2 q \varepsilon_{s} \varepsilon_{0} N\right)^{1 / 2}\left(\delta / \varepsilon_{i} \varepsilon_{0}\right)$, which can be readily evaluated assuming typical values for the doping level extracted from the slope of the Mott-Schottky analysis $N \sim 10^{16} \mathrm{~cm}^{-3}$ (see Supporting Information) and the dielectric permittivity of the organic bulk $\varepsilon_{\mathrm{s}} \sim 3$. In our case, the correction results in negligible values of $<0.1 \mathrm{meV}$, which allows us to safely identify $V_{\mathrm{fb}}=V_{\mathrm{bb}}$ and $\Delta=\Delta^{\prime}$. These last equalities support the procedure of determining the band bending from the intercept of the Mott-Schottky plot. In terms of the device operation, it entails that forward applied voltages $V_{\mathrm{app}}<V_{\mathrm{fb}}$ reduce the band bending, while they leave unaltered the voltage drop at the dipole layer; that is, there is no significant change in the interface charge $Q_{i s}$.

Experimental Determination of Flat-Band Values and Dipole Layers. Equation 6 predicts the behavior observed in Figure 3. A linear fitting results in $S=0.08$ with $g_{\text {is }}=$ $4 \times 10^{14} \mathrm{~cm}^{-2} \mathrm{eV}^{-1}$ calculated using eq 7. The low value encountered for the parameter $S$ indicates a significant degree of Fermi level pinning, and this implies a large shift for the metal Fermi level displacement along the polymer band gap in the process of contact equilibration. From eq 6 , an estimation of CNL can also be evaluated from the linear fit. This last calculation gives a value $E_{\mathrm{CNL}}=4.7 \mathrm{eV}$. The position of the Fermi level corresponding to the p-doped organic blend is approximated by means of an expression $E_{\mathrm{Fp}} \approx E_{\mathrm{HOMO}}^{\mathrm{D}}-k_{\mathrm{B}} T \ln \left(N_{\mathrm{HOMO}} / p_{0}\right)$, which assumes Boltzmann statistics for the hole occupancy of the HOMO levels. The density of states at the HOMO level is taken as $N_{\text {Hомо }} \sim 10^{20} \mathrm{~cm}^{-3}$, and the background hole density caused by the doping as extracted from the Mott-Schottky analysis results in $p_{0}=N \sim 5 \times$ $10^{15} \mathrm{~cm}^{-3}$ (see Supporting Information and Table 1 ). Under these assumptions, one arrives at $E_{\mathrm{Fp}} \approx 4.95 \mathrm{eV}$, a value that is located at deeper energies with respect to $E_{\mathrm{CNL}}$. The position reached by the metal Fermi level calculated as $E_{\mathrm{Fm}}=E_{\mathrm{Fp}}-q V_{\mathrm{fb}}$ indicates that the interface states exercise a large influence to locate it near CNL (see Table 1). Because the Au work function lies at larger values than $E_{\mathrm{CNL}}$ (see Figure $1 \mathrm{~A}$ ), the calculation of the parameter $S$ has not considered this value.

The previous analysis leads us to view the cathode contact energetics as drawn in Figure 1C. The main portion of the work function mismatch, $\phi_{c}-\phi_{s}$, is not equilibrated by the space charge region (majority carrier depletion zone) but through the voltage drop at the interface dipole layer. The question that arises at
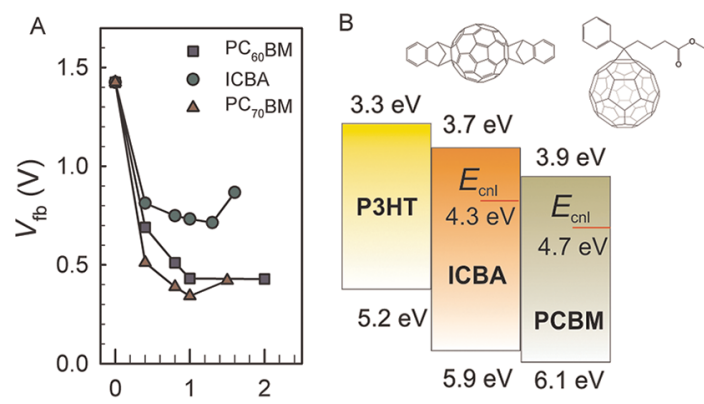

Fullerene: $\mathrm{P} 3 \mathrm{HT}$ ratio

Figure 4. (A) Variation of the flat-band voltage with the blend ratio for different fullerenes. Lines are used to guide the eyes. (B) Energy diagram indicating the position of the $\mathrm{CNL}$ and the corresponding fullerene energy levels.

this stage concerns the nature of the interface states responsible for the dipole layer formation. Also noticeable is the deep location of the charge-neutrality level $E_{\mathrm{CNL}}=4.7 \mathrm{eV}$ with respect to the vacuum level. We propose here that the negative charge on the organic side of the interface is originated by reduced fullerene molecules attached to the metal contact and energetically located within the effective band gap IDOS. In the vicinity of the metal, the energy spread of fullerene LUMO levels produced by the inherent disorder of the organic blend is expected to be even larger than that encountered in fullerene bulk molecules.

The assumption of a constant $g_{\text {is }}$ used in the derivation of eq 6 is an oversimplification of the actual IDOS, in any case, the low value found for the parameter $S$ points to a large interfacial DOS at lower energies which effectively pins the metal Fermi level at $\sim 4.6 \mathrm{eV}$ with respect to the vacuum level (see Table 1). This energy approximately coincides with the position extracted for CNL. We remark here that this value is also in agreement with the observed saturation in the Fermi level of PCBM layers on coated substrates $^{33}$ as derived from ultraviolet (UPS) and X-ray (XPS) photoelectron spectroscopy.

Dependence of Flat-Band Values and Dipole Layers on the Composition of the Blend. In order to gain further insight into the origin of the interface states and reinforce our conjecture which links IDOS to reduced fullerene molecules, we have performed a series of experiments varying the blend composition. Three different fullerene acceptors have been tested: namely, ICBA, $P C_{60} B M$, and $P C_{70} B M$, with the aim of checking the effect of the acceptor LUMO level shift on the cathode energetics. In addition, the P3HT:fullerene blend ratio is varied with the aim to experimentally determine the sensitivity of the contact energetics to the organic composition. The $j-V$ characteristics corresponding to these experiments are shown in Supporting Information. In these experiments, the cathode metal is kept unaltered to low work function Ca. Summarized in Figure $4 \mathrm{~A}$ is the variation in $V_{\mathrm{fb}}$ as a function of the 


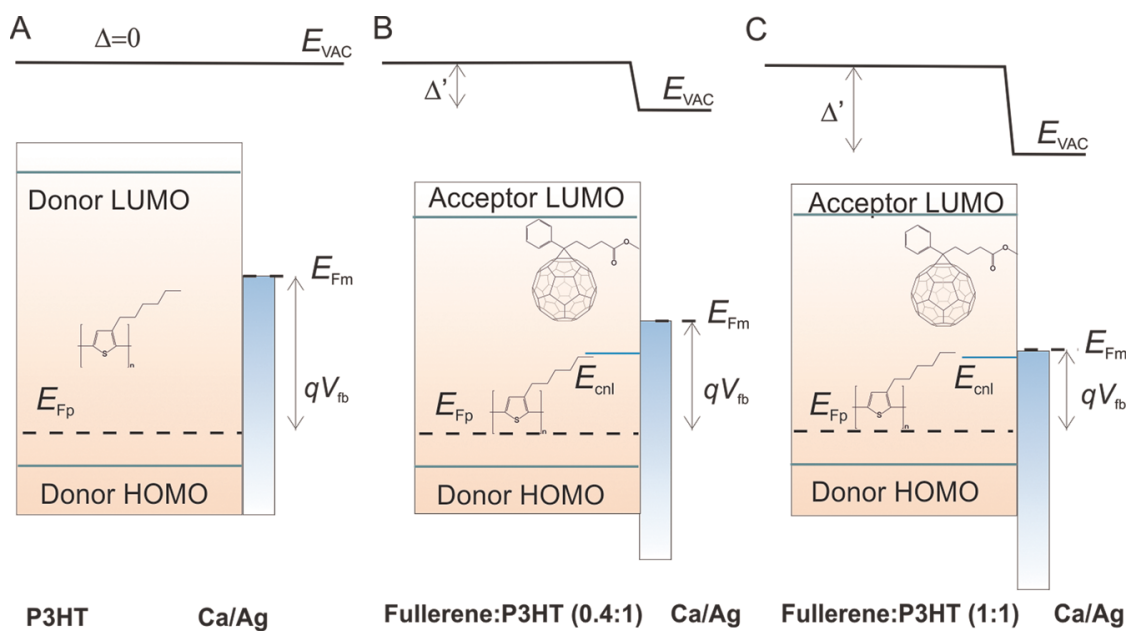

Figure 5. Diagram of the cathode contact in flat-band conditions. (A) Flat vacuum level and large $V_{\mathrm{fb}}$ because of the dipole layer absence in the case of the active layer comprising only polymer. (B) Case of low content of fullerene: the dipole layer accommodates a small portion of the work function offset, and the metal Fermi level is situated above the CNL. (C) For larger fullerene content, $E_{\mathrm{Fm}}$ is practically pinned at $\mathrm{CNL}$, and a large dipole layer appears.

fullerene:P3HT blend ratio for three different fullerenes used. The first experimental finding to note is the value obtained for devices prepared with only polymer $V_{\mathrm{fb}}=$ $1.5 \mathrm{~V}$, which is in rather good agreement with the expected large offset between the metal and organic layer work functions, $\phi_{\mathrm{c}}-\phi_{\mathrm{s}} \approx 1.9 \mathrm{eV}$, as drawn in Figure $5 \mathrm{~A}$. Here the cathode contact ability to form a dipole layer is very limited because no acceptor molecules are present at the interface. The band bending is then the principal mechanism to equilibrate the contact in accordance to the Schottky limit.

By incrementing the amount of fullerene molecules in the device, $V_{\mathrm{fb}}$ steeply decreases (Figure $4 \mathrm{~A}$ ), as expected when the amount of acceptor states attached to the cathode metal increases, then enlarges the dipole voltage drop. The effect of incrementing the fullerene content of the blend is illustrated in Figure 5. These diagrams correspond to flat-band conditions. Lower coverage of the contact interface by fullerene molecules (small IDOS) has an important effect on the contact equilibration. By reducing $g_{\text {is }}$ (larger $S$ ), one might envisage an increment in band bending as inferred from eq 1 . When the fullerene amount is increased, interface coverage reaches a maximum, then reduces $V_{\mathrm{fb}}$ and enlarges the dipole voltage offset $\Delta$. Interestingly, $V_{\mathrm{fb}}$ reaches a plateau at blend ratio approaching 1:1. As drawn in Figure 5C, the metal Fermi level approaches the charge-neutrality level for a blend ratio of $\sim 1: 1$. We would not expect significant changes in the position of CNL by using different content of the same fullerene, although this mainly depends on the actual IDOS distribution (Figure 5B,C).

Another key aspect of this investigation is to explore the effect of the fullerene energetics on the cathode equilibration. By changing the type of fullerene, it is expected that the IDOS varies, as well. Reported LUMO energy levels for ICBA and $\mathrm{PC}_{60} \mathrm{BM}$
(3.74 and $3.91 \mathrm{eV}$, respectively) show a shift of $0.17 \mathrm{eV}$ as calculated from cyclic voltammetry. ${ }^{34}$ These energy levels are shown in Figure $4 \mathrm{~B}$ as well as those corresponding to $\mathrm{P} 3 \mathrm{HT}$. By examining Figure $4 \mathrm{~A}$, one can observe that both $\mathrm{PC}_{60} \mathrm{BM}$ - and $\mathrm{PC}_{70} \mathrm{BM}$-based devices yield a flat-band voltage plateau for blend ratio $(\sim 1: 1)$ of $V_{\mathrm{fb}} \sim 0.4 \mathrm{~V}$. As explained previously, the metal Fermi level approximately coincides with that calculated for the charge-neutrality level $E_{\mathrm{CNL}} \approx 4.7 \mathrm{eV}$. Since the same trend is observed when ICBA is used as fullerene acceptor (with a different value for the flat-band plateau $V_{\mathrm{fb}} \sim 0.7 \mathrm{~V}$ ), one can infer that CNL is shifted to lower energy positions. In this case, we obtain a value of $E_{\mathrm{CNL}} \approx 4.3 \mathrm{eV}$. Such downward displacement is in qualitative agreement with the general IDOS energy shift caused by the fullerene LUMO level offset (Figure 4B). Because ICBA has different properties as compared with $\mathrm{PCBM}$ molecules, it might also be possible for the same blend ratio $(\sim 1: 1)$ that a reduced amount of ICBA molecules covers the cathode interface. This would imply that the increase in $V_{\mathrm{fb}}$ is explained by differences in concentration rather than molecular energetics. We show in Supporting Information, AFM and cross-section SEM images of P3HT:PCBM and P3HT:ICBA films, which exhibit very similar morphology. These experiments reinforce the idea of an energy-related origin for the differences observed in $C-V$ analysis between PCBM- and ICBA-based devices. It is then inferred from the previous discussion that band bending and dipole layer strength are extremely dependent on the amount and nature of fullerene molecules attached to the metal cathode.

Effect of Cathode Interlayers. Cathode electron-selective interlayers are commonly used to both enhance energy level alignment and to prevent holes from crossing the contact. The inclusion of these interlayers is supposed to largely modify the interface electronic 
TABLE 2. Photovoltaic Parameters and Parameters Extracted from the Capacitance Mott-Schottky Analysis of Thin P3HT: Fullerene Solar Cells with Different Cathode Interlayers

\begin{tabular}{|c|c|c|c|c|c|c|c|c|}
\hline $\begin{array}{c}\text { Р3НT:PC }{ }_{60} \text { BM } \\
(100 \mathrm{~nm})\end{array}$ & $\begin{array}{c}j_{\mathrm{sc}} \\
\left(\mathrm{mA} \mathrm{cm}{ }^{-2}\right)\end{array}$ & $\begin{array}{l}V_{o c} \\
(V)\end{array}$ & FF & $\begin{array}{l}\text { PCE } \\
(\%)\end{array}$ & $\begin{array}{c}N \\
\left(10^{15} \mathrm{~cm}^{-3}\right)\end{array}$ & $\begin{array}{l}V_{\mathrm{fb}} \\
(V)\end{array}$ & $\begin{array}{l}E_{\mathrm{Fm}} \\
(\mathrm{eV})\end{array}$ & $\begin{array}{c}\Delta \\
(\mathrm{eV})\end{array}$ \\
\hline $\mathrm{Ca}$ & 8.72 & 0.62 & 0.62 & 3.4 & 10 & 0.42 & 4.53 & 1.66 \\
\hline $\mathrm{BCP} / \mathrm{Ca}$ & 8.00 & 0.63 & 0.63 & 3.2 & 25 & 0.44 & 4.51 & 1.64 \\
\hline LiF/Al & 7.73 & 0.60 & 0.62 & 3.0 & 20 & 0.44 & 4.51 & 0.36 \\
\hline $\mathrm{C}_{60} / \mathrm{Ca}$ & 7.68 & 0.61 & 0.60 & 2.8 & 47 & 0.48 & 4.47 & 1.60 \\
\hline
\end{tabular}

structure which enhances the device performance. We have studied here the effect of $\mathrm{LiF}^{35}$ bathocuproine $\mathrm{BCP}^{36}$ and evaporated $\mathrm{C}_{60}$ thin layers on interfacial properties as displayed in the Mott-Schottky characteristics. Details on the deposition methods of these interlayers can be obtained in Supporting Information. In these experiments, thinner active P3HT:PCBM layers $(100 \mathrm{~nm})$ have been used which has allowed increasing the cell performance (PCE > 3\%) as observed by comparing Tables 1 and 2 for Ca cathode contact. In Figure $6, j-V$ curves and Mott-Schottky plots are shown. It is observed that $V_{\mathrm{fb}}$ extracted from the intercept (Figure 6b) is located around $0.4 \mathrm{~V}$ despite the use of different interlayers. This observation indicates that the $\mathrm{CNL}$ underpins the contact at $E_{\mathrm{Fm}} \approx 4.5 \mathrm{eV}$, in agreement with results presented in the previous sections. Interestingly, $V_{\mathrm{fb}}$ is observed to be highly active layer thickness-independent: $0.39 \mathrm{~V}$ for $350 \mathrm{~nm}$ thick film and $0.42 \mathrm{~V}$ for $100 \mathrm{~nm}$ thick film in the case of Ca cathode. This finding reinforces the idea of the crucial role played by interface states on the local equilibration of the contact.

We finally remark that the equilibration at the cathode interface is the key mechanism to understand the photovoltaic effect. ${ }^{21}$ When the solar device operates in high forward (or large illumination intensities under open-circuit conditions), the rise of the bulk electron Fermi level induces an increment within the occupancy of fullerene DOS states. A good connection between the internal and the cathode metal Fermi
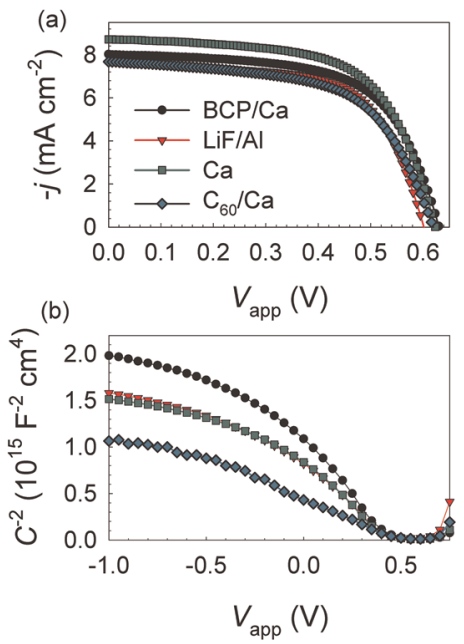

Figure 6. (a) Current-voltage characteristics of devices with structure ITO/PEDOT:PSS/P3HT:PCBM/cathode contact as indicated. (b) Mott-Schottky plots from which the p-doping level $N$ and flat-band voltage $V_{\mathrm{fb}}$ are extracted. Note the similar value obtained from the voltage intercept around $0.4 \mathrm{~V}$.

level is now assured by the interface states identified here using capacitance methods.

\section{CONCLUSION}

We have provided a detailed analysis of the cathode energy equilibration of bulk-heterojunction solar cells. The Fermi level mismatch established between the cathode metal and the organic layer is equilibrated by the vacuum level offset and split into two contributions: spatially extended band bending in the organic bulk and voltage drop at the interface dipole layer. The Mott-Schottky analysis allows us to extract both the flat-band voltage (band bending) and the p-doping density. It has been experimentally observed that the dipole layer withstands a large part of the total Fermi level mismatch when the blend ratio approaches $\sim 1: 1$. Interface states are related to fullerene reduced molecules covering the metal contact. We have concluded that the dipole contribution, and consequently the band bending, is highly sensitive to the fullerene interface DOS.

\section{METHODS}

Device Fabrication. Polymer solar cells were fabricated with a standard sandwich structure of ITO/PEDOT:PSS/P3HT:fullerene/ metal/Ag and $9 \mathrm{~mm}^{2}$ of active area. PEDOT:PSS was spin-coated in air at $5500 \mathrm{rpm}$ for $30 \mathrm{~s}$ onto an ITO-coated glass substrate (10 $\Omega / \mathrm{sq})$, film thickness of $\sim 35 \mathrm{~nm}$. The substrates were heated at $120{ }^{\circ} \mathrm{C}$ for $10 \mathrm{~min}$ to remove traces of water and were transferred to a glovebox equipped with a thermal evaporator. The P3HT:fullerene layer was deposited at speeds of $1200 \mathrm{rpm}$ for $30 \mathrm{~s}$ followed by a slow drying of the film in a Petri dish. At this point, samples were thermally annealed at $130^{\circ} \mathrm{C}$ for $20 \mathrm{~min}$. Sequential evaporation of the interfacial layer or metal was carried out at a base pressure of $3 \times 10^{-6} \mathrm{mbar}$ under study and was terminated by evaporation of a protective Ag layer (100 nm). Devices were encapsulated by using a pressure-sensitive glue (polyisobutylene, Oppanol B 12 SFN from BASF) and a glass microscope slide. Samples were then taken out of the glovebox for device characterization. A detailed description of the structures can be found in Supporting Information.

Device Characterization. Current density-voltage and capacitance measurements were carried out by illumination with a $1.5 \mathrm{G}$ illumination source $\left(1000 \mathrm{~W} \mathrm{~m}^{-2}\right)$ using an Abet Sun 2000 solar simulator. The light intensity was adjusted with a calibrated Si solar cell. Capacitance was recorded by applying a small voltage perturbation $(20 \mathrm{mV} \mathrm{rms})$ at $100 \mathrm{~Hz}$. Measurements were carried out in the dark at different bias voltage to extract the capacitance-voltage characteristics. These measurements were performed with Autolab PGSTAT-30 equipped with a frequency analyzer module. 
Conflict of Interest: The authors declare no competing financial interest.

Acknowledgment. We thank financial support from Ministerio de Educacion y Ciencia under project HOPE CSD200700007 (Consolider-Ingenio 2010), and Generalitat Valenciana (Prometeo/2009/058, ACOMP/2009/056 and ACOMP/2009/ 095). L.F.M. acknowledges Conselho Nacional de Desenvolvimento Científico e Tecnológico (CNPq) for a grant (Processo: 201380/2010-2).

Supporting Information Available: The capacitance-voltage analysis performed to extract the background charge density and the flat-band voltage is explained, along with examples of $j-V$ curves and fabrication conditions. Analysis of active layer topography and device cross section is also provided. This material is available free of charge via the Internet at http:// pubs.acs.org.

\section{REFERENCES AND NOTES}

1. Steim, R.; Koglera, F. R.; Brabec, C. J. Interface Materials for Organic Solar Cells. J. Mater. Chem. 2010, 20, 2499-2512.

2. Ratcliff, E. L.; Zacher, B.; Armstrong, N. R. Selective Interlayers and Contacts in Organic Photovoltaic Cells. J. Phys. Chem. Lett. 2011, 2, 1337-1350.

3. Po, R.; Carbonera, C.; Bernardia, A.; Camaioni, N. The Role of Buffer Layers in Polymer Solar Cells. Energy Environ. Sci. 2011, 4, 285-310.

4. Yip, H.-L.; Hau, S. K.; Baek, N. S.; Ma, H.; Jen, A. K.-Y. Polymer Solar Cells That Use Self-Assembled-Monolayer-Modified ZnO/Metals as Cathodes. Adv. Mater. 2008, 20, 2376-2382.

5. Seo, J. H.; Gutacker, A.; Sun, Y.; Wu, H.; Huang, F.; Cao, Y.; Scherf, U.; Heeger, A. J.; Bazan, G. C. Improved HighEfficiency Organic Solar Cells via Incorporation of a Conjugated Polyelectrolyte Interlayer. J. Am. Chem. Soc. 2011, 133, 8416-8419.

6. Wang, D. H.; Moon, J. S.; Seifter, J.; Jo, J.; Park, J. H.; Park, O. O.; Heeger, A. J. Sequential Processing: Control of Nanomorphology in Bulk Heterojunction Solar Cells. Nano Lett. 2011, 11, 3163-3168.

7. Schmidt-Hansberg, B.; Sanyal, M.; Klein, M. F. G.; Pfaff, M.; Schnabel, N.; Jaiser, S.; Vorobiev, A.; Müller, E.; Colsmann, A.; Scharfer, P.; et al. Moving through the Phase Diagram: Morphology Formation in Solution Cast Polymer-Fullerene Blend Films for Organic Solar Cells. ACS Nano 2011, 5, 8579-8590.

8. Ishii, H.; Sugiyama, K.; Ito, E.; Seki, K. Energy Level Alignment and Interfacial Electronic Structures at Organic/Metal and Organic/Organic Interfaces. Adv. Mater. 1999, 11, 605-625.

9. Crispin, X.; Geskin, V.; Crispin, A.; Cornil, J.; Lazzaroni, R.; Salaneck, W. R.; Bredas, J.-L. Characterization of the Interface Dipole at Organic/Metal Interfaces. J. Am. Chem. Soc. 2002, 124, 8131-8141.

10. Hwang, J.; Wan, A.; Kahn, A. Energetics of Metal-Organic Interfaces: New Experiments and Assessment of the Field. Mater. Sci. Eng. R 2009, 64, 1-31.

11. Braun, S.; Salaneck, W. R.; Fahlman, M. Energy-Level Alignment at Organic/Metal and Organic/Organic Interfaces. Adv. Mater. 2009, 21, 1450-1472.

12. Salaneck, W. R.; Seki, K.; Kahn, A.; Pireaux, J.-J. Conjugated Polymer and Molecular Interfaces. Science and Technology for Photonic and Optoelectronic Applications; Marcel Dekker: New York, 2002.

13. Bardeen, J. Surface States and Rectification at a Metal Semi-conductor Contact. Phys. Rev. 1947, 71, 717-727.

14. Vázquez, H.; Flores, F.; Oszwaldowski, R.; Ortega, J.; Pérez, R.; Kahn, A. Barrier Formation at Metal-Organic Interfaces: Dipole Formation and the Charge Neutrality Level. Appl. Surf. Sci. 2004, 234, 107-112.

15. Vázquez, H.; Gao, W.; Flores, F.; Kahn, A. Energy Level Alignment at Organic Heterojunctions: Role of the Charge Neutrality Level. Phys. Rev. B 2005, 71, 041306(R).

16. Baldo, M. A.; Forrest, S. R. Interface-Limited Injection in Amorphous Organic Semiconductors. Phys. Rev. B 2001, $64,085201$.
17. Ishii, H.; Hayashi, N.; Ito, E.; Washizu, Y.; Sugi, K.; Kimura, Y.; Niwano, M.; Ouchi, Y.; Seki, K. Kelvin Probe Study of Band Bending at Organic Semiconductor/Metal Interfaces: Examination of Fermi Level Alignment. Phys. Status Solidi A 2004, 201, 1075-1094.

18. Abdou, M. S. A.; Orfino, F. P.; Son, Y.; Holdcroft, S. Interaction of Oxygen with Conjugated Polymers: Charge Transfer Complex Formation with Poly(3-alkylthiophenes). J. Am. Chem. Soc. 1997, 119, 4518-4524.

19. Hoshino, S.; Yoshida, M.; Uemura, S.; Kodzasa, T.; Takada, N.; Kamata, T.; Yase, K. Influence of Moisture on Device Characteristics of Polythiophene-Based Field-Effect Transistors. J. Appl. Phys. 2004, 95, 5088-5093.

20. Liang, Z.; Nardes, A.; Wang, D.; Berry, J. J.; Gregg, B. A. Defect Engineering in $\pi$-Conjugated Polymers. Chem. Mater. 2009, 21, 4914-4919.

21. Bisquert, J.; Garcia-Belmonte, G. On Voltage, Photovoltage and Photocurrent in Bulk Heterojunction Organic Solar Cells. J. Phys. Chem. Lett. 2011, 2, 1950-1964.

22. Lee, J.; Kong, J.; Kim, H.; Kang, S.-O.; Lee, K. Direct Observation of Internal Potential Distributions in a Bulk Heterojunction Solar Cell. Appl. Phys. Lett. 2011, 99, 243301.

23. Nishi, T.; Kanai, K.; Ouchi, Y.; Willis, M. R.; Seki, K. Evidence for the Atmospheric p-Type Doping of Titanyl Phthalocyanine Thin Film by Oxygen Observed as the Change of Interfacial Electronic Structure. Chem. Phys. Lett. 2005, 414, 479-482.

24. Fabregat-Santiago, F.; Garcia-Belmonte, G.; Mora-Seró, I.; Bisquert, J. Characterization of Nanostructured Hybrid and Organic Solar Cells by Impedance Spectroscopy. Phys. Chem. Chem. Phys. 2011, 13, 9083-9118.

25. Boix, P. P.; Garcia-Belmonte, G.; Muñecas, U.; Neophytou, M.; Waldauf, C.; Pacios, R. Determination of Gap Defect States in Organic Bulk Heterojunction Solar Cells from Capacitance Measurements. Appl. Phys. Lett. 2009, 95, 233302.

26. Morfa, A. J.; Nardes, A. M.; Shaheen, S. E.; Kopidakis, N.; van de Lagemaat, J. Time-of-Flight Studies of Electron-Collection Kinetics in Polymer:Fullerene Bulk-Heterojunction Solar Cells. Adv. Funct. Mater. 2011, 21, 2580-2586.

27. Nalwa, K. S.; Mahadevapuram, R. C.; Chaudhary, S. Growth Rate Dependent Trap Density in Polythiophene-Fullerene Solar Cells and Its Implications. Appl. Phys. Lett. 2011, 98, 093306.

28. Li, J. V.; Nardes, A. M.; Liang, Z.; Shaheen, S. E.; Gregg, B. A.; Levi, D. H. Simultaneous Measurement of Carrier Density and Mobility of Organic Semiconductors Using Capacitance Techniques. Org. Electron. 2011, 12, 1879-1885.

29. Cowley, A. M.; Sze, S. M. Surface States and Barrier Height of Metal Semiconductor Systems. J. Appl. Phys. 1965, 65, 3212-3220.

30. Cowley, A. M. Depletion Capacitance and Diffusion Potential of Gallium Phosphide Schottky-Barrier Diode. J. Appl. Phys. 1966, 37, 3024-3032.

31. Garcia-Belmonte, G.; Bolink, H. J.; Bisquert, J. Capacitcance-Voltage Characteristics of Organic Light-Emitting Diodes Varying the Cathode Metal: Implications for Interfacial States. Phys. Rev. B 2007, 75, 085316.

32. Bisquert, J.; Garcia-Belmonte, G.; Munar, A.; Sessolo, M.; Soriano, A.; Bolink, H. J. Band Unpinning and Photovoltaic Model for P3HT-PCBM Organic Bulk Heterojunctions under Illumination. Chem. Phys. Lett. 2008, 465, 57-62.

33. Xu, Z.; Chen, L. M.; Chen, M.-H.; Li, G.; Yang, Y. Energy Level Alignment of Poly(3-hexylthiophene):[6,6]-Phenyl-C61Butyric Acid Methyl Ester Bulk Heterojunction. Appl. Phys. Lett. 2009, 95, 013301.

34. He, Y.; Chen, H.-Y.; Hou, J.; Li, Y. Indene-C60 Bisadduct: A New Acceptor for High-Performance Polymer Solar Cells. J. Am. Chem. Soc. 2010, 132, 1377-1382.

35. Shaheen, S. E.; Brabec, C. J.; Sariciftci, N. S.; Padinger, F.; Fromherz, T.; Hummelen, J. C. $2.5 \%$ Efficient Organic Plastic Solar Cells. Appl. Phys. Lett. 2001, 78, 841-843.

36. Chang, C.-C.; Lin, C.-F.; Chiou, J.-M.; Ho, T.-H.; Tai, Y.; Lee, J.-H.; Chen, Y.-F.; Wang, J.-K.; Chen, L.-C.; Chen, K.-H. Effects of Cathode Buffer Layers on the Efficiency of Bulk-Heterojunction Solar Cells. Appl. Phys. Lett. 2010, 96, 263506. 